\section{Insomnie bei Nahrungsmittelallergie}

Helga Peter

Marburg, Deutschland

\section{Synonyme}

Nahrungsmittelallergie-bedingte Insomnie

\section{Englischer Begriff}

food allergy insomnia

\section{Definition}

Es handelt sich um einen mittlerweile historischen Begriff für Ein- und Durchschlafstörungen, die als Reaktion auf eine Nahrungsmittelunverträglichkeit auftreten, sehr häufig bei Kuhmilchallergie.

In der ICSD-R (International Classification of Sleep Disorders - Revised) von 1997 wurde eine Insomnie bei Nahrungsmittelallergie als extrinsische Dyssomnie gelistet, eine Kategorie, die in der ICSD-2 von 2005 aufgegeben wurde. Dort wurde sie unter der Hauptkategorie Insomnien als Insomnie durch Medikamente oder Substanzen klassifiziert. Nach > „ICSD-3“ (2014) kann eine derartige Störung als Andere Insomnie diagnostiziert werden.

Siehe auch $>$,Diagnostische Klassifikationssysteme“. 